\title{
Ground-based lidar and microwave radiometry synergy for high vertical resolution absolute humidity profiling
}

\author{
María Barrera-Verdejo $^{1}$, Susanne Crewell ${ }^{1}$, Ulrich Löhnert ${ }^{1}$, Emiliano Orlandi $^{1}$, and Paolo Di Girolamo ${ }^{2}$ \\ ${ }^{1}$ Institut für Geophysik und Meteorologie, Universität zu Köln, Cologne, Germany \\ ${ }^{2}$ Scuola di Ingegneria, Universita degli Studi della Basilicata, Potenza, Italy
}

Correspondence to: María Barrera-Verdejo (mbarrera@smail.uni-koeln.de)

Received: 11 February 2016 - Published in Atmos. Meas. Tech. Discuss.: 23 February 2016

Revised: 1 July 2016 - Accepted: 1 July 2016 - Published: 24 August 2016

\begin{abstract}
Continuous monitoring of atmospheric humidity profiles is important for many applications, e.g., assessment of atmospheric stability and cloud formation. Nowadays there are a wide variety of ground-based sensors for atmospheric humidity profiling. Unfortunately there is no single instrument able to provide a measurement with complete vertical coverage, high vertical and temporal resolution and good performance under all weather conditions, simultaneously. For example, Raman lidar (RL) measurements can provide water vapor with a high vertical resolution, albeit with limited vertical coverage, due to sunlight contamination and the presence of clouds. Microwave radiometers (MWRs) receive water vapor information throughout the troposphere, though their vertical resolution is poor. In this work, we present an MWR and RL system synergy, which aims to overcome the specific sensor limitations. The retrieval algorithm combining these two instruments is an optimal estimation method (OEM), which allows for an uncertainty analysis of the retrieved profiles. The OEM combines measurements and a priori information, taking the uncertainty of both into account. The measurement vector consists of a set of MWR brightness temperatures and RL water vapor profiles. The method is applied to a 2-month field campaign around Jülich (Germany), focusing on clear sky periods. Different experiments are performed to analyze the improvements achieved via the synergy compared to the individual retrievals. When applying the combined retrieval, on average the theoretically determined absolute humidity uncertainty is reduced above the last usable lidar range by a factor of $\sim 2$ with respect to the case where only RL measurements are used. The analysis in terms of degrees of freedom per signal reveal that most information is gained above the usable lidar range, especially
\end{abstract}

important during daytime when the lidar vertical coverage is limited. The retrieved profiles are further evaluated using radiosounding and Global Position Satellite (GPS) water vapor measurements. In general, the benefit of the sensor combination is especially strong in regions where Raman lidar data are not available (i.e., blind regions, regions characterized by low signal-to-noise ratio), whereas if both instruments are available, RL dominates the retrieval. In the future, the method will be extended to cloudy conditions, when the impact of the MWR becomes stronger.

\section{Introduction}

Highly resolved, accurate and continuous measurements of water vapor are required for a deeper understanding of many atmospheric phenomena (Stevens and Bony, 2013). Specifically, processes on short timescales such as convection, cloud formation or boundary layer turbulence are challenging due to their high associated water vapor variability, which is difficult to capture with one instrument alone (Steinke et al., 2015). In order to overcome this limitation, the scientific community has started merging different data from several instruments in the last 15 years.

Some examples of ground-based synergies have been proposed by Stankov (1998), Löhnert et al. (2004), Furumoto et al. (2003) or Bianco et al. (2005) and Delanoe and Hogan (2008) for satellite applications. In the present paper, the synergy between ground-based Raman lidar (RL) and microwave radiometer (MWR) instruments is described. Both instruments present some advantages and disadvantages and, by bringing them together in an optimal and new retrieval al- 
gorithm, it is possible to overcome some of the disadvantages of the single devices and enhance their benefits.

Water vapor RL systems provide humidity profiles with high vertical resolution. For this reason, such lidars have become a powerful tool in active ground-based observations over recent years. New retrieval algorithms optimally exploiting the information content have been developed (Sica and Haefele, 2015; Povey et al., 2014; Sica and Haefele, 2016). However, the RL techniques alone show some drawbacks, which hinder the operational application. For example, ground-based RL cannot provide information above and within optically thick clouds, as the radiation emitted by the lidar is severely attenuated once the laser beam reaches a liquid layer within the cloud. Moreover, daytime measurements are affected by solar background radiation, which strongly reduces the vertical extent of the profile. The continuous and effective detection of the weak Raman signals demands a robust and stable alignment of the receiving system. Daytime operation requires the use of powerful lasers whose continuous operation though possible, is technically demanding (Dinoev et al., 2013; Brocard et al., 2013). Additionally, RL needs to be regularly calibrated. This calibration is usually performed based on the use of radiosounding data, which presents some caveats. First, the balloon might measure a different air volume due to its drift. Second, it implies rather high costs, both instrumental as well as human resources. The calibration of the lidar is a key point that still stimulates new solutions (Foth et al., 2015). In addition, lidar data from the lowest atmospheric layers typically cannot be used, due to the presence of a blind region (or zero overlap region (ZOR)) associated with the overlap function (OVF) of the RL.

The MWR allows automated continuous data acquisition and is a robust operational instrument (Rose et al., 2005), measuring unattended in a 24/7 mode. Brightness temperature measurements at different frequencies allow the determination of humidity and temperature profiles. In contrast to $\mathrm{RL}$, the instrument offers a limited vertical resolution in the retrieved atmospheric profiles, especially in the higher layers of the atmosphere (i.e., above an altitude of $1 \mathrm{~km}$ ) (Löhnert et al., 2007), but performs best for measurements close to the ground, where the lidar data are missing. The MWR also provides accurate integrated quantities such as integrated water vapor (IWV) or liquid water path (LWP) (Crewell and Löhnert, 2003; Löhnert and Crewell, 2003). The calibration of this instrument is performed with internal and external references with known temperature (hot load-cold load) or by observing the atmosphere under different elevation angles (i.e., sky tipping) (Maschwitz et al., 2013). An advantage of the MWR is its capability of measuring in almost all weather conditions (also cloudy cases) except for rainy scenarios, where the received signal must be discarded in most of the cases.

A method to combine RL and MWR was already proposed by Han et al. (1997), where the authors developed a two-stage algorithm to derive atmospheric water vapor profiles. In the first stage, a Kalman filtering algorithm was applied using surface in situ and RL measurements. In the second stage, a statistical inversion technique was applied to combine the Kalman retrieval (used as prior information, not as observations) with the integrated water vapor of a two-channel MWR and climatological data. Their method showed that the synergy of these two sensors compensates for the individual sensors' drawbacks. A continuation of this work was carried out by Schneebeli (2009) who, still following the Kalman filter two-stage configuration, extends this approach to also temperature profiles.

The method described in this document is a new approach based on an optimal estimation method (OEM), an iterative optimal and physically consistent method that allows uncertainty assessment and provides the most probable estimated atmospheric state together with its uncertainty description. The aim of this study is to combine the information provided by the two instruments in an OEM to retrieve atmospheric water vapor profiles. Note that this flexible framework allows the retrieval of temperature once corresponding $\mathrm{RL}$ and MWR data are available. The method was applied to the 2month dataset collected during HOPE $\left(\mathrm{HD}(\mathrm{CP})^{2}\right.$ Observational Prototype Experiment), where a multitude of groundbased remote sensing instruments for the investigation of boundary layer and cloud processes were operated (Steinke et al., 2015; Behrendt et al., 2015; Foth et al., 2015). Here we focus on clear sky cases and absolute humidity profiles. A description of the method is presented in Sect. 3. Section 4 describes the results when the OEM is applied to a case study, whereas Sect. 5 evaluates the OEM when applied to the 2-month period of HOPE. Finally, Sect. 6 summarizes the results and provides an outlook.

\section{Observations: HOPE}

In this study we make use of the data collected during HOPE (HD(CP)2 Observational Prototype Experiment), which was a major field campaign in North Rhine-Westphalia, Germany, from April to June 2013. One main goal of HOPE was to provide information on subgrid variability (i.e., of water vapor) and microphysical properties on scales smaller than $1 \mathrm{~km}$, which corresponds to the horizontal resolution of state-ofthe-art operational mesoscale models. During the measurement period, three supersites were operating that were distributed within the 5-10 km surroundings of Forschungszentrum Jülich, Germany (50.905, 6.411944). Each supersite was composed of a rich variety of remote sensing instruments such as cloud radar, lidar and microwave radiometer instruments. A large set of more than 200 radiosondes (RSs) was launched only $4 \mathrm{~km}$ away from the JOYCE (Jülich ObservatorY for Cloud Evolution) site and at least twice a day.

At the permanent supersite JOYCE (Löhnert et al., 2014), measurements by the University of Basilicata Raman lidar 
system (BASIL) and an MWR were carried out, and auxiliary data from other instruments are available.

\subsection{BASIL}

The Raman lidar system BASIL (Di Girolamo et al., 2009; Di Girolamo et al., 2012) is an active instrument detecting the elastic and Raman backscattered radiation from atmospheric constituents. BASIL includes a Nd:YAG laser emitting pulses at its fundamental wavelength, and its second and third harmonics: 355,532 and $1064 \mathrm{~nm}$, respectively, at a repetition rate of $20 \mathrm{~Hz}$. For the purpose of water vapor profiling, Raman scattering of the $355 \mathrm{~nm}$ beam is used because of the higher cross section with respect to other wavelengths. The average power emitted at $355 \mathrm{~nm}$ is $10 \mathrm{~W}$. Nevertheless, other transmitting wavelengths could also be used for water vapor detection, as reported by Althausen et al. (2000). The receiver is built around a larger telescope in Newtonian configuration (45 cm diameter primary mirror) and two smaller telescopes (5 $\mathrm{mm}$ diameter lenses). The larger telescope is primarily dedicated to the collection of the Raman signals, i.e., the water vapor and molecular nitrogen roto-vibrational Raman signals, at 407.5 and $386.7 \mathrm{~nm}$, respectively, which are used to estimate the water vapor mixing ratio profiles.

Signal selection is performed by means of narrowband interference filters, whose specifications were reported in Di Girolamo et al. (2004) and Di Girolamo et al. (2009). Sampling of the Raman signals is performed by means of transient recorders with double-signal acquisition mode (i.e., both analog, A/D conversion and digital, photon counting). Depending on the application, water vapor mixing ratio profiles can be derived with different vertical and temporal resolutions. These two parameters can be traded off to improve measurement precision. For the purposes of this study, the lidar products are characterized by a vertical resolution of $30 \mathrm{~m}$ and a temporal resolution of $5 \mathrm{~min}$. Because of the absence of overlap between the laser beam and receiver field of view, there is a blind region in the lower altitudes. Consequently, vertical profiles of water vapor mixing ratio typically start at $150-180 \mathrm{~m}$ above ground. Humidity profiles extend vertically up to different altitudes during daytime and nighttime depending on the altitude where the signal gets completely extinguished. For water vapor, considering a vertical/temporal resolution of $30 \mathrm{~m} / 5 \mathrm{~min}$, this typically takes place around $4-5 \mathrm{~km}$ during daytime and around $12 \mathrm{~km}$ during the night. The different ranges result from the additional noise due to solar contamination during daytime.

During HOPE, BASIL was calibrated based on the comparison with the radiosondes launched approximately $4 \mathrm{~km}$ away from the instrument. A mean calibration coefficient was estimated by comparing BASIL and radiosonde data. Every clear sky radiosonde coincident with BASIL measurements (60 in total) is compared to the lidar profile in an altitude region with an extent of $1 \mathrm{~km}$ above the boundary layer. We choose this region to minimize the air mass differences related to the distance between the lidar station and the radiosonde launch facility station. For every profile comparison, a value for the calibration constant is calculated. From these 60 values, we calculate the mean value and use it as the calibration constant for the complete period of HOPE. The standard deviation of the mean calibration coefficient from the single values does not exceed $5 \%$.

In addition to the calibration constant uncertainty, other smaller systematic uncertainty sources might affect the water vapor measurements. For example, an additional uncertainty $(<1 \%)$ may be considered related to the use of narrowband filters, the temperature dependence of $\mathrm{H}_{2} \mathrm{O}$ Raman scattering and the thermal sensitivity of the filters (Whiteman, 2003). Further, an additional $1 \%$ may be associated with the determination of the differential transmission term at the water vapor and molecular nitrogen Raman wavelengths (Whiteman, 2003). These sources of uncertainty, in principle negligible, are not taken into account for the calculations in our algorithm.

The statistical uncertainty of the water vapor mixing ratio is calculated based on the application of the Poisson statistics (Di Girolamo et al., 2004) and varies for each range bin. Providing a profile with $5 \mathrm{~min}$ time resolution and $30 \mathrm{~m}$ vertical grid, the statistical uncertainty affecting water vapor mixing ratio measurements for nighttime operation is typically smaller than $2 \%$ up to $3 \mathrm{~km}$ and smaller than $20 \%$ up to $9 \mathrm{~km}$. For daytime operation, it is typically smaller than $40 \%$ up to $3 \mathrm{~km}$ and smaller than $100 \%$ up to $4.5 \mathrm{~km}$.

Note, the operation of BASIL has not been continuous during HOPE. The instrument collected a total of $430 \mathrm{~h}$ of measurements distributed over 44 days, which represents $30 \%$ of the whole HOPE period.

\subsection{MWR}

The microwave radiometer profiler HATPRO (Rose et al., 2005) was manufactured by Radiometer Physics $\mathrm{GmbH}$, Germany (RPG), as a network-suitable microwave radiometer allowing retrieval of liquid water path (LWP) and integrated water vapor (IWV) at high temporal resolution (1 s) (Crewell and Löhnert, 2003). It is a passive MWR that measures radiation expressed as brightness temperature in two frequency bands (Rose et al., 2005). The seven channels of the $\mathrm{K}$ band contain information about the vertical profile of humidity through the pressure broadening of the optically thin $22.235 \mathrm{GHz} \mathrm{H}_{2} \mathrm{O}$ line. This band also provides the information for determining LWP as emission by liquid water increases with frequency. The seven channels of V-band contain information on the vertical profile of temperature resulting from the homogeneous mixing of $\mathrm{O}_{2}$ throughout the atmosphere (Löhnert and Maier, 2012). For temperature, retrieval improvement can be obtained by including off-zenith observations under the assumption of horizontal homogeneity; however for water vapor profiling, only zenith observations are beneficial (Löhnert et al., 2009). 
The absolute calibration of the instrument is performed roughly every 6 months, taking a cold and a hot load as reference, which are assumed to be ideal black bodies. The cold black body is a liquid-nitrogen-cooled load at approximately $77 \mathrm{~K}$ that is attached externally to the radiometer box. This standard, together with an internal ambient black body load inside the radiometer, is used for the absolute calibration procedure (Maschwitz et al., 2013). In addition, a calibration by tip-curve observations can be performed for the K-band channels using observations at different elevation angles (Turner et al., 2007). The reliability of sky tipping calibrations strongly depends on how good the assumption of a horizontally stratified atmosphere is. Further details on the calibration procedures of the instrument can be found in Maschwitz et al. (2013).

The temporal resolution of this instrument is higher than for the RL: it is able to provide one measurement every 1$3 \mathrm{~s}$. Thus, a temporal adaptation to the lidar time resolution is performed, averaging MWR measurements in 5 min intervals. A major drawback of MWR water vapor and temperature profile retrievals is the limited vertical resolution. Typically, only two pieces of independent information for water vapor profiles are contained in the measurements, whereby three-four are obtained for the temperature profile (Löhnert et al., 2009).

\section{Method}

\subsection{Optimal estimation method}

An optimal estimation method is applied which allows the state of the atmosphere and its associated uncertainty to be estimated. Using this scheme requires a set of measurements (with their uncertainty specification), a forward model, which relates the atmospheric state to the instrument measurements and some a priori information. In the following, a short description of the scheme is presented. More details can be found in Rodgers (2000).

Given the moderately nonlinear nature (Rodgers, 2000) of our problem, the iterative equation applied to find the best atmospheric state estimate is

$$
\begin{aligned}
& \boldsymbol{x}_{i+1}=\boldsymbol{x}_{\mathrm{a}}+\left(\mathbf { S } _ { \mathrm { a } } \mathbf { K } _ { i } ^ { T } ( \mathbf { K } _ { i } \mathbf { S } _ { \mathrm { a } } \mathbf { K } _ { i } ^ { T } + \mathbf { S } _ { \epsilon } ) ^ { - 1 } \left[\boldsymbol{y}-\mathbf{F}\left(\boldsymbol{x}_{i}\right)\right.\right. \\
& \left.\left.\quad+\mathbf{K}_{i}\left(\boldsymbol{x}_{i}-\boldsymbol{x}_{\mathrm{a}}\right)\right]\right),
\end{aligned}
$$

where $\boldsymbol{x}_{i}$ is a vector containing the atmospheric state at the iteration $i$. The observation vector $\boldsymbol{y}$ contains the brightness temperatures (TBs) from the MWR and the profile of the mixing ratio from the lidar. The term $\boldsymbol{x}_{\mathrm{a}}$ represents the a priori information of the atmosphere, in our case, coming from radiosondes. $\mathbf{S}_{\mathrm{a}}$ and $\mathbf{S}_{\epsilon}$ are the covariance matrices of the prior and observation uncertainties, respectively. $\mathbf{F}\left(\boldsymbol{x}_{i}, b\right)$ is the forward model applied to the state vector $\boldsymbol{x}_{i}$, and depends on the model parameters $b$. For simplicity, it will be referred to as $\mathbf{F}\left(\boldsymbol{x}_{i}\right)$ in the following. The forward model output lies in the observation space. The term $\mathbf{K}$ represents the Jacobian, which can be understood as the response of the observation vector when a perturbation is performed in the atmospheric state vector (Eq. 2):

$\mathbf{K}_{i}=\frac{\partial \mathbf{F}\left(\boldsymbol{x}_{i}\right)}{\partial \boldsymbol{x}_{i}}$.

The iterative equation described in Eq. (1) finds the most optimal atmospheric state $\boldsymbol{x}_{\mathrm{op}}$. Convergence of the solution is reached once the convergence criterion is fulfilled; i.e., the difference between the forward model applied to the atmospheric state at iterations $n$ and $n+1$ is 1 order of magnitude smaller than the estimated error. To evaluate this difference we must scale the change in the solution by its estimated error, leading to

$$
\begin{aligned}
d_{i}^{2} & =\left(\mathbf{F}\left(\boldsymbol{x}_{i+1}\right)-\mathbf{F}\left(\boldsymbol{x}_{i}\right)\right)^{T}\left(\mathbf{S}_{\epsilon}\left(\mathbf{K S}_{\mathrm{a}} \mathbf{K}^{T}+\mathbf{S}_{\epsilon}\right) \mathbf{S}_{\epsilon}\right)^{-1}\left(\mathbf{F}\left(\boldsymbol{x}_{i+1}\right)\right. \\
& \left.-\mathbf{F}\left(\boldsymbol{x}_{i}\right)\right)<m / 10,
\end{aligned}
$$

where $m$ is the number of elements in the observation vector. An uncertainty estimation of the solution $\mathbf{S}_{\mathrm{op}}$ is calculated via

$\mathbf{S}_{\mathrm{op}}=\mathbf{S}_{\mathrm{a}}-\mathbf{S}_{\mathrm{a}} \mathbf{K}^{T}\left(\mathbf{S}_{\epsilon}+\mathbf{K S}_{\mathrm{a}} \mathbf{K}^{T}\right)^{-1} \mathbf{K S}_{\mathrm{a}}$,

where $\mathbf{K}$ is the Jacobian calculated in the last iteration. From $S_{\mathrm{op}}$, the theoretical error (in $\mathrm{kg} \mathrm{m}^{-3}$ ) associated to each altitude of the retrieved profile $\boldsymbol{x}_{\mathrm{op}}$ is calculated as the square root of the main diagonal elements in $\mathbf{S}_{\mathrm{op}}$. The word "theoretical" emphasizes that it is an a posteriori estimate, and not a direct difference to a given reference. It is also possible to estimate the information content of the result. The degrees of freedom (DOF) of a profile represent the number of independent pieces of information in the signal. They can be calculated as the trace of the matrix in Eq. (5):

$\mathbf{A}_{\mathrm{ker}}=\mathbf{S}_{\mathrm{a}} \mathbf{K}^{T}\left(\mathbf{S}_{\epsilon}+\mathbf{K S}_{\mathrm{a}} \mathbf{K}^{T}\right)^{-1} \mathbf{K}$,

where $\mathbf{A}_{\text {ker }}$ is the averaging kernel. This matrix is very important to describe the information content, as it describes the subspace of the state space in which the retrieval must lie. Its diagonal elements can be seen as a measure of the number of degrees of freedom per discrete altitude level. The reciprocal denotes the number of levels per degree of freedom and can be interpreted as a measure of resolution. The vertical resolution $\Delta \boldsymbol{z}$ is thus defined as the range of heights covered divided by the number of independent quantities measured:

$\boldsymbol{\Delta} z=\frac{\boldsymbol{\delta} z}{\operatorname{diag}\left(\mathbf{A}_{\mathrm{ker}}\right)}$,

where $\delta z$ is the vertical spacing grid for the retrieval. It is important to note the difference between the vertical discretization of the retrieved profile and the quantification of the effective vertical resolution $\boldsymbol{\Delta} z$. 


\subsection{A priori: $x_{\mathrm{a}}$ and $\mathrm{S}_{\mathrm{a}}$}

The a priori information is calculated from the set of radiosondes launched during HOPE. A total of 217 sondes have been considered. Generally, at least two of them are available for every day of the campaign, typically one around noon and the other at midnight. From these data, the average profile of absolute humidity $\boldsymbol{q}$, in $\mathrm{kg} \mathrm{m}^{-3}$, is calculated to represent the a priori knowledge, together with its standard deviation $s_{q}$. This profile is used as $\boldsymbol{x}_{\mathrm{a}}$ in the algorithm described by Eq. (1).

For the same set of radiosondes, the correlation (corr) and covariance (cov) matrices are calculated according to Wilks (2006), to describe the relation of absolute humidity between two different altitude levels. We can define $\boldsymbol{q}$ that represents absolute humidity as a function of the altitude:

$\boldsymbol{q}=\left[q_{1}, q_{2}, \ldots q_{k}\right]$,

with $k$ being the total number of altitudes in the retrieval vertical grid. Therefore, the corr and cov matrices have a dimension of $k \times k$, calculated with the formula:

$$
\begin{aligned}
& \operatorname{corr}_{\boldsymbol{q}_{a}, \boldsymbol{q}_{b}}=\frac{\operatorname{cov}\left(\boldsymbol{q}_{a}, \boldsymbol{q}_{b}\right)}{s_{q_{\mathrm{a}}} s_{q_{b}}} \\
& \quad=\frac{\frac{1}{n-1} \sum_{i=1}^{n}\left[\left(q_{a_{i}}-\overline{\boldsymbol{q}}_{a}\right)\left(q_{b_{i}}-\overline{\boldsymbol{q}}_{b}\right)\right]}{\left[\frac{1}{n-1} \sum_{i=1}^{n}\left(q_{a_{i}}-\overline{\boldsymbol{q}}_{a}\right)^{2}\right]^{\frac{1}{2}}\left[\frac{1}{n-1} \sum_{i=1}^{n}\left(q_{b_{i}}-\overline{\boldsymbol{q}}_{b}\right)^{2}\right]^{\frac{1}{2}}},
\end{aligned}
$$

where $i$ denotes each radiosonde, with a total of $n=217$. The parameter $\overline{\boldsymbol{q}}$ is the mean vertical profile of absolute humidity, and $a$ and $b$ are indices for all the different $k$ altitudes.

Both covariance and correlation matrices have been calculated as in Eq. (8). The first is needed in the retrieval algorithm as input $\left(\mathbf{S}_{\mathrm{a}}\right)$, the second because it better illustrates the relations between water vapor at different altitudes in the atmosphere. The correlation matrix (Fig. 1) illustrates how the absolute humidity at a certain altitude is correlated with the one at other altitudes, from ground to $10 \mathrm{~km}$. The values for the correlation are strongest close to the main diagonal, but decrease quickly for off-diagonal terms. In the lowest $1-2 \mathrm{~km}$ there is a higher correlation, because of the well mixed conditions in the boundary layer. The result is similar to previous studies (Ebell et al., 2013).

\subsection{Observations: $y$ and $S_{\epsilon}$}

The measurement vector $\boldsymbol{y}$ is composed of the TBs from the MWR and the water vapor mixing ratio (WVMR) profile from the RL. We choose the TBs to be part of the measurement vector instead of the MWR-derived profile of humidity in order to give the OEM the freedom to distribute the water vapor information to those heights where the lidar provides no information. In addition, for future applications, it allows us to extend our algorithm to simultaneous, physically consistent retrievals of temperature and liquid water. WVMR is

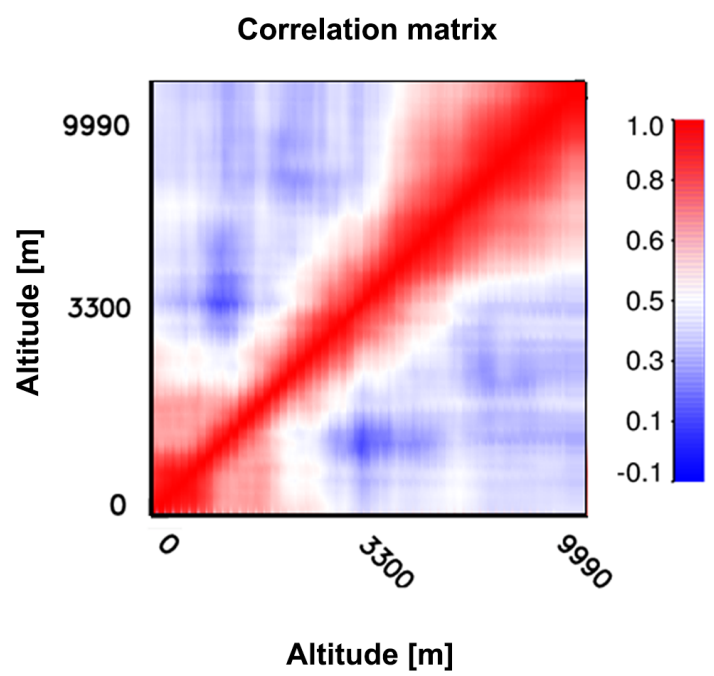

Figure 1. Correlation matrix derived from 217 radiosondes launched during HOPE. Correlation is shown for absolute humidity as a function of the altitude (from 0 to $10 \mathrm{~km}$ above the ground).

used as the lidar measurement (with uncertainties given in Sect. 2.1), which allows the use of a complex lidar forward operator to be avoided.

The size of $\boldsymbol{y}$ is variable; since it depends on the number of values, the lidar is able to measure at every given time interval. A lidar mixing ratio profile $\left(\mathrm{kg} \mathrm{kg}^{-1}\right)$, together with its statistical uncertainty, is provided with a vertical resolution of $30 \mathrm{~m}$, starting from $180 \mathrm{~m}$ (See Sect. 2.1). Below this altitude, the lidar detectors cannot be interpreted in a meaningful way due to the lack of overlap of the emitted and received beams. Due to decreasing signal-to-noise ratio (SNR) with height, one must determine the altitude up to which the lidar data can be considered meaningful. This altitude range has been defined via the relative uncertainty of the WVMR, which is calculated at each altitude as the ratio between the uncertainty and the measurement itself. When this value is larger than $100 \%$, the data are considered too noisy and are discarded. Care is required when applying this threshold because possible random peaks in the lidar uncertainty can lead to a filtering of too many points. Therefore, a running average is performed on the data with a $300 \mathrm{~m}$ window size in the vertical. This smoothed profile is only used to select the clipping altitude for the RL data. The $100 \%$ uncertainty altitude is reached at different heights depending on the weather situation or night/day periods. Typically it was found around $3-4 \mathrm{~km}$ during daytime and around $7-8 \mathrm{~km}$ during nighttime measurements.

In effect, the observation vector $\boldsymbol{y}$ is composed of $t+m$ elements, and $\mathbf{S}_{\epsilon}$ is a matrix with dimensions $(t+m, t+m) ; m$ is the number of altitudes where the lidar measurements have sufficient signal-to-noise ratio, and $t$ is the number of TBs. Seven brightness temperatures are used for the retrieval of absolute humidity. Note that within the retrieval procedure, 
TBs from the MWR are used directly in the measurement vector, while an atmospheric state variable (WVMR) is used from the lidar to complete the measurement vector that only requires a conversion of humidity units.

The error covariance matrix associated with the MWR measurement (with dimensions $7 \times 7$, is obtained empirically by calculating the covariance for the different channels, while constantly viewing an ambient black-body target with known temperature. The diagonal elements represent the covariance of each channel with itself, typically with values around the noise level $(\sim 0.25 \mathrm{~K})$. The off-diagonal elements represent the covariance between the measurements of different channels. Because the channels share some electronic components inside the instrument, the off-diagonal elements cannot be considered zero, but typically show values 1 order of magnitude smaller than the main diagonal.

The part of $\mathbf{S}_{\epsilon}$ corresponding to the RL (dimension ( $m \times$ $m$ ) is defined as a diagonal matrix containing only the random uncertainty at every altitude. This definition implies no correlation between measurements at different heights. This simplification in the error covariance matrix has also been considered by other authors (Wulfmeyer et al., 2006; Dunbar et al., 2014; Adam et al., 2015). The $\mathbf{S}_{\epsilon}$ elements corresponding to the correlation between RL and MWR measurements have been set to zero because no correlation is expected among measurement uncertainty of two separate instruments.

\subsection{Forward models (FMs)}

The forward model for the lidar is straightforward because in our retrieval approach we consider WVMR as part of the measurements vector. Therefore, the lidar FM for water vapor simply performs the conversion from absolute humidity to mixing ratio. However, the implementation of a more complex lidar forward model, e.g., the approach implemented by Sica and Haefele (2016), could be considered in future studies. The FM for the MWR involves a radiative transfer model (Löhnert et al., 2004). It considers emission and absorption of radiation by gases in the atmosphere but neglects scattering, which can be ignored for all atmospheric particles except for rain droplets. The model divides the atmosphere into layers and calculates the optical thickness and absorption coefficients at each level. From these values, and applying the radiative transfer Eq. (9) (Janssen, 1993), the TBs are calculated:

$$
\begin{aligned}
& \mathrm{TB}_{\text {ground }}=\mathrm{TB}_{\cos } \exp (-\tau) \\
& \quad+\int_{0}^{\infty} T(s) \alpha(s) \exp \left(-\int_{0}^{s} \alpha\left(s^{\prime}\right) \mathrm{d} s^{\prime}\right) \mathrm{d} s,
\end{aligned}
$$

where $\tau$ is the optical depth of the whole atmospheric column (opacity), $\alpha$ is the absorption coefficient $\left(\mathrm{m}^{-1}\right)$ and $\mathrm{TB}_{\cos }$ is the cosmic background radiation (approx. $2.7 \mathrm{~K}$ ).
The retrieval vertical grid is defined for every profile. It varies, as well as the observation vector, depending on the amount of available lidar information for every given profile. In the atmospheric regions where lidar data are available, the vertical grid of the retrieval product is $30 \mathrm{~m}$ (same as the lidar). Above the point where the RL signal is lost, and since the MWR cannot provide such high resolution, the algorithm retrieves only one value every $1 \mathrm{~km}$.

\section{Application of the OEM: case study}

\subsection{Single profile retrieval}

In a first approach, the OEM is implemented for the combination of the two instruments to retrieve atmospheric absolute humidity. The setup is designed such that the OEM can work with input from a single instrument as well. This aspect allows us to compare the performance of each sensor working alone in contrast to the combination of the both. In the following, we demonstrate the algorithm presenting the results corresponding to 24 April at 11:00 UTC, where a collocated RS is used only as reference (Fig. 2). The a priori profile is the prior atmospheric knowledge (Sect. 3.2), and also the starting point (first guess) for the algorithm iteration.

At first, we only introduce the portion of profile in the OEM where RL data are considered to be valid (i.e., from $180 \mathrm{~m}$ to $2.5 \mathrm{~km}, \sim 77$ layers), not taking into account the MWR yet. The result of the algorithm is a complete profile from the ground up to $10 \mathrm{~km}$. In the region with lidar availability, the result is strongly constrained by the lidar observations, since the associated uncertainties are very small (on the order of $0.5 \mathrm{~g} \mathrm{~m}^{-3}$ ). In the regions with no lidar data, the profile is completed with the information provided by the a priori profile and the a priori covariance matrix. Second, if only the seven TBs of the MWR are introduced in the OEM, a very smooth profile is obtained. This is because the seven frequencies do not provide enough information to distinguish fine vertical structures: MWR can only provide $\sim 2$ DOF per profile, as already mentioned in Sect. 2.2. Therefore, the a priori profile plays a dominant role in defining the vertical structures. Finally, the output profile for the RL and MWR combination is strongly constrained to the RL observations from $180 \mathrm{~m}$ to $2.5 \mathrm{~km}$. Outside this region, the profile is completed based on the information provided by the TBs and the a priori.

The OEM uncertainty of the combined retrieval is calculated as the square root of the main diagonal elements in $\mathbf{S}_{\mathrm{op}}$ (see Eq. 4). The uncertainty is small in the region where there are RL data available $\left(\sim 0.5 \mathrm{~g} \mathrm{~m}^{-3}\right)$, but it increases with altitude, as to be expected (Fig. 2). It is also slightly larger close to the ground $\left(\sim 1 \mathrm{~g} \mathrm{~m}^{-3}\right)$, due to the absence of lidar data. Throughout the profile, the combined retrieval uncertainty is smaller than the "only-RL" and "only-MWR" ones. (see also Sect. 5.3 for detailed uncertainty statistics). 


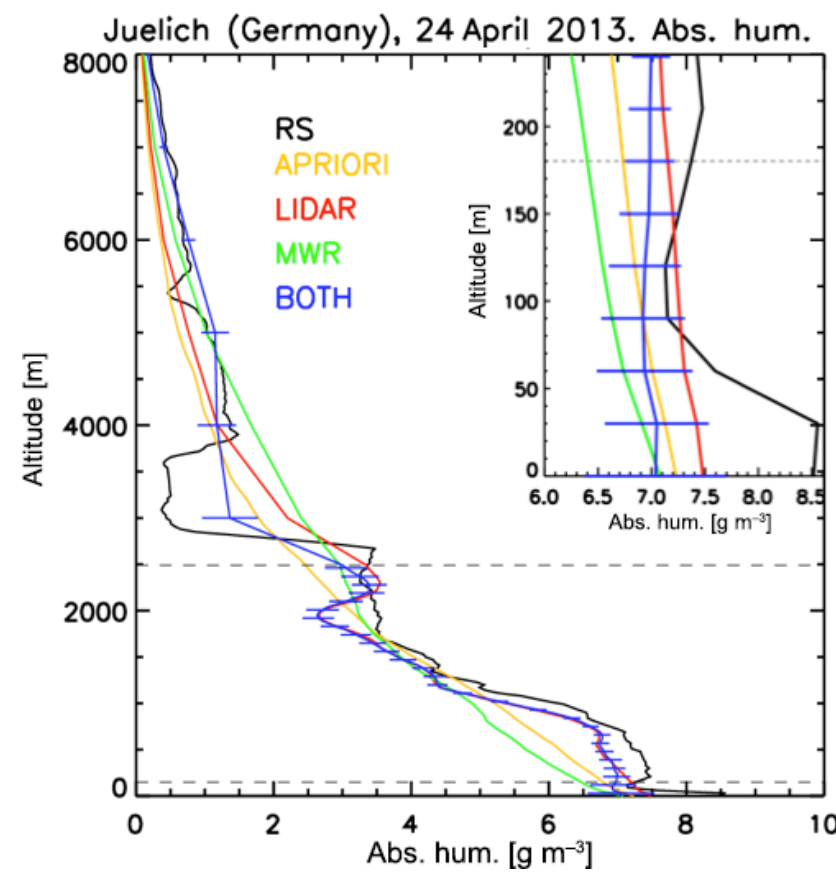

Figure 2. Absolute humidity profiles for a priori (yellow), onlyRL (red), only-MWR (green) and MWR+RL (blue). The horizontal blue lines correspond to the theoretical retrieval error for the MWR+RL case. The RS is used as reference (black). The dashed horizontal gray lines enclose the region where the lidar data are used. The inset is a zoom for the region close to the ground, between 0 and $250 \mathrm{~m}$.

The profile obtained with the RL-MWR combination best fits the RS (shown as reference), launched at the same time $4 \mathrm{~km}$ away. The combined retrieval reveals absolute humidity values closer to the radiosonde at $3 \mathrm{~km}$ than single instrument retrievals. This is due to both the additional microwave radiometer observations as well as propagated lidar information (via the a priori covariance matrix). It is interesting to pay attention to the lower part of the atmosphere, close to the ground. In Fig. 2, a zoom from 0 to $250 \mathrm{~m}$ is shown. Due to the missing RL information below $180 \mathrm{~m}$, the RLMWR combination tends to the MWR values close to the ground, but quickly approaches the lidar, as soon as the first RL values are available. One can see that the lowest values of the RS are $1-1.5 \mathrm{~g} \mathrm{~m}^{-3}$, more humid than the rest of the profiles. This might be explained by the fact that the sonde was launched under different local environmental conditions: while the instruments site is located inside the research center, the RS is launched in an open field area. In addition, the venting of the RS is not optimal in the lowest $100 \mathrm{~m}$. These could cause slight differences in the comparisons close to the ground, but should not be a problem in the free troposphere.

We can additionally evaluate the quality of our retrieval by calculating the effective vertical resolution. Figure 3 presents the vertical resolution $\Delta z$ calculated with Eq. (6) for the three different retrievals on the 24 April 2013, at 11:00 UTC. The

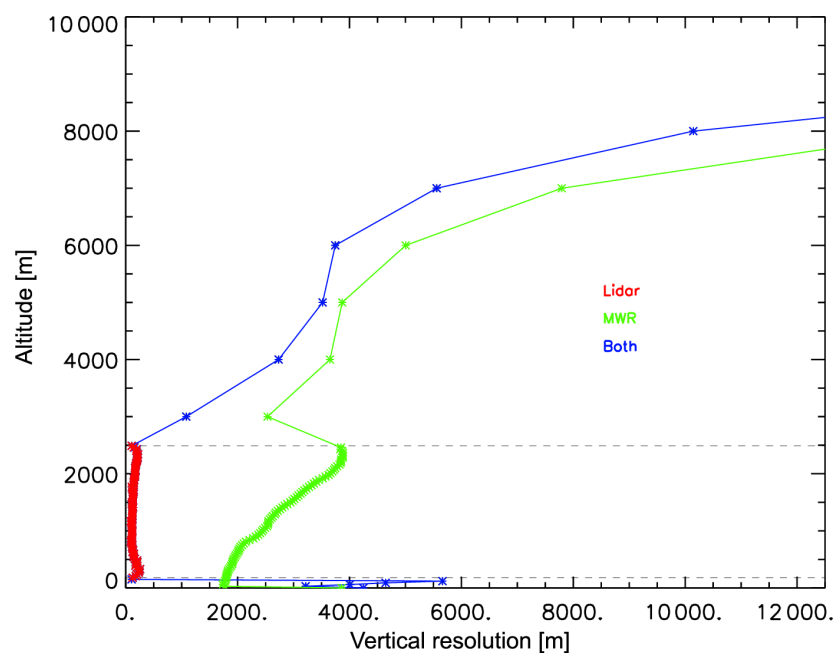

Figure 3. Vertical resolution for the only-RL (red), only-MWR (green) and MWR+RL (blue). The dashed lines enclose the area where RL data have been considered.

results nicely show the improvements of the MWR+RL combination. In the region where RL is available (from $180 \mathrm{~m}$ to $2.5 \mathrm{~km})$, the only-RL resolution is very high $(\sim 100-300 \mathrm{~m})$. However, outside this region, the vertical resolution for onlyRL becomes infinite, because the diagonal elements of the averaging kernels tend to zero. The only-MWR resolution is always coarser: up to $2.5 \mathrm{~km}$ it presents values 1 order of magnitude larger than the other two cases. Nevertheless, the advantage of the MWR is that the instrument provides information throughout the complete profile. Finally, the MWR+RL case presents the best vertical resolution. It adopts similar values as the only-RL resolution when RL is available, and improves the resolution by $\sim 1-2 \mathrm{~km}$ compared to the only-MWR case throughout the rest of the profile. Since the solution is strongly constrained by the lidar observations between $180 \mathrm{~m}$ and $2.5 \mathrm{~km}$, the additional information contained in the MWR observations is now mainly distributed in the region above the $2.5 \mathrm{~km}$.

\subsection{Time series}

The combined retrieval is now applied to more than one profile. An example of this is shown in Fig. 4, which presents a time series of absolute humidity on 4 May 2013 during HOPE, for RL, MWR and MWR+RL. Note that the plots are presented for the lowest $4 \mathrm{~km}$ because above this altitude, no significant changes of humidity occur. In addition, and in order to appreciate visually the added value of synergy, the native retrievals of MWR and RL are shown, i.e., the RL mixing ratio converted into absolute humidity and the absolute humidity profiles calculated from the MWR with a simple statistical retrieval based on a quadratic regression method (a multivariate regression scheme based on an extensive radiosonde data set from DeBilt). 
(a)

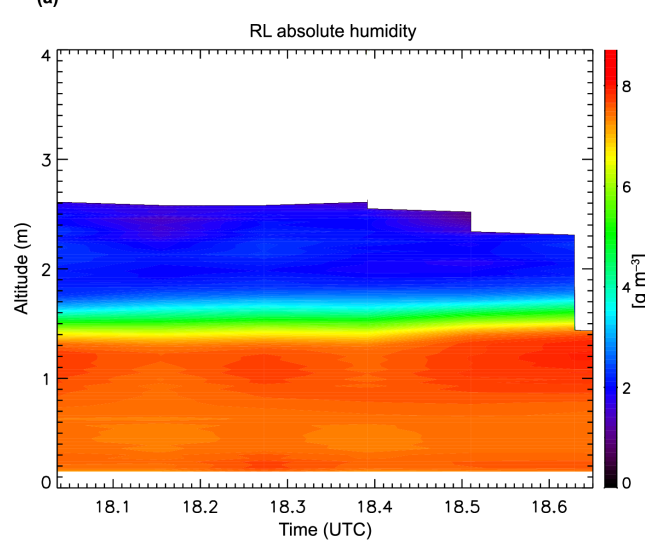

(b)

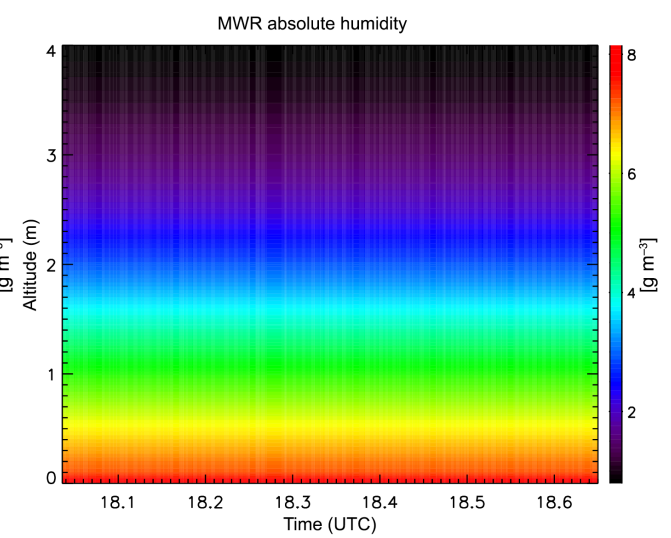

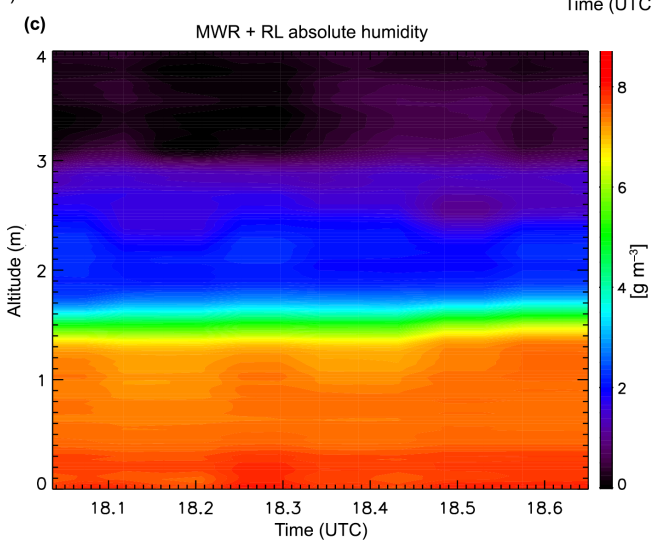

Figure 4. From left to right and top to bottom, absolute humidity $\left(\mathrm{g} \mathrm{m}^{-3}\right)$ time series of RL, MWR statistical retrieval and MWR+RL retrieval.

One can see clearly how the RL zero overlap region does not allow any information from the lowest $180 \mathrm{~m}$ to be received. In addition, the lidar signal is strongly affected by the background daytime radiation from around $2.5 \mathrm{~km}$ above. Note that following the explanation in Sect. 3.3, the RL data, whose relative error is larger than $100 \%$, are discarded. In contrast to the RL, the information provided by the MWR is continuous regardless the background radiation. Nevertheless, the vertical resolution of the MWR profiles is extremely poor compared to the RL.

The MWR+RL time series reveals a successful synergy between RL and MWR, making use of the TB and a priori information to complete the profile where RL measurements are not available (i.e., in the blind region below $180 \mathrm{~m}$ and at regions of too high a lidar noise level).

\section{Application of the OEM: statistics over HOPE}

The absolute humidity algorithm has been applied to all the clear sky periods with simultaneous availability of MWR and RL. The MWR measured continuously, so this selection is restricted to lidar availability. There are 4201 lidar profiles in total ( $30 \%$ of the total campaign). Of these, 717 profiles are considered as clear sky (around $17 \%$ of the total). Of the clear sky profiles, the convergence of the OEM is found in $95.8 \%$ of the cases, that is, 687 profiles. In the rest of the cases, the convergence is not reached because the algorithm cannot find a profile which is simultaneously consistent with the measurements of the two instruments and the a priori information, within their uncertainties.

\subsection{Integrated water vapor}

Another key atmospheric parameter that we can evaluate after applying the OEM is the IWV. The independent measurements of IWV from the Global Position Satellite (GPS) ground station (Bevis et al., 1992) can be used to assess the quality of the retrieval products. In Fig. 5a, the time series of the IWV during HOPE is presented. The continuous IWV signal from GPS measurements is shown together with the IWV from the joint retrieval, which is only available during clear sky events. IWV reveals strong fluctuations with values between 5 and $29 \mathrm{~kg} \mathrm{~m}^{-2}$ during HOPE, and therefore this period is well suited for evaluation studies.

Figure 5b quantitatively compares the three OEM retrieval cases (combined retrieval, MWR and only-RL) against the GPS signal. Note that a comparison with the original lidar 

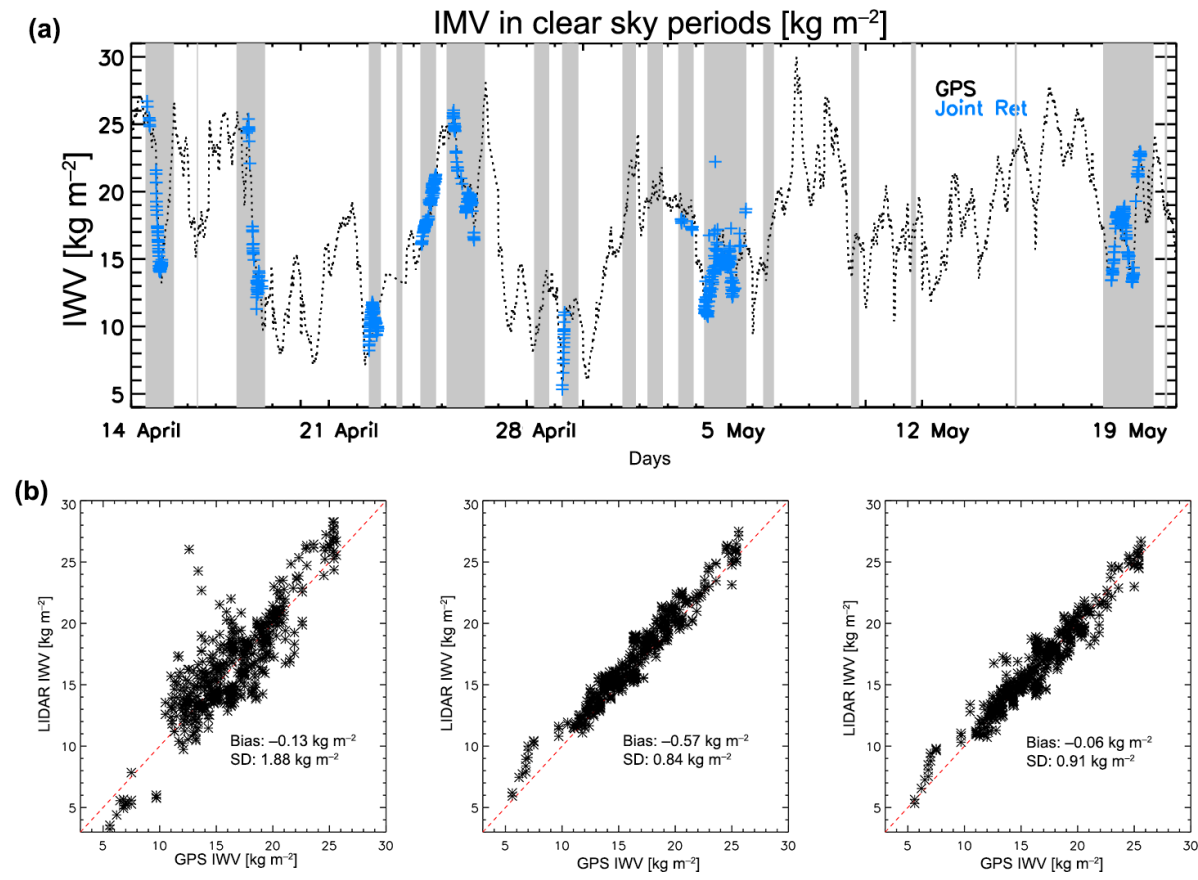

Figure 5. (a) Time series of IWV during the whole HOPE period from the continuous GPS signal (black) and the one calculated from the joint retrieval, which is available only in clear sky cases (blue). Shaded areas represent the RL availability. (b) Scatter plot for the three cases: only Raman lidar, only MWR and the joint retrieval (from left to right), against the GPS.

data before processing in the OEM is not sensible, since the lidar lacks information in the lowest atmosphere (due to incomplete overlap) and also above the altitude where the SNR is too large. A sensible comparison is only carried out after OEM processing because these retrieval results provide full profiles in all three cases.

Figure $5 \mathrm{~b}$ also shows the values for the bias and the standard deviation (in $\mathrm{kg} \mathrm{m}^{-2}$ ) for all the cases. The values are small in all situations and lie inside the GPS uncertainty of $1-2 \mathrm{~kg} \mathrm{~m}^{-2}$ (Gendt et al., 2004) and the MWR product of $\sim 0.5-1 \mathrm{~kg} \mathrm{~m}^{-2}$ (Steinke et al., 2015). While the only-MWR case presents a negative bias of $\sim 0.5 \mathrm{~kg} \mathrm{~m}^{-2}$, the inclusion of the RL in the RL+MWR case corrects this bias, reducing it 1 order of magnitude. The combination of the two instruments and the only-MWR case presents similar standard deviations, whereas the only-RL case presents twice as large a standard deviation in comparison to the other two cases. This results give us confidence that the developed OEM water vapor profiles are well constrained with respect to the integrated value.

\subsection{Comparison to RS}

As explained above, the retrieval grid of each profile depends on how much data from the RL can be taken into account, which will depend on the atmospheric conditions, day/night, background noise, etc. In order to clearly assess the benefits of the sensor synergy, a different retrieval strategy is used for the subsequent tests: the algorithm is applied using only the
RL profiles up to a fixed altitude in order to retrieve all profiles using the same vertical grid. Thus, all RL profiles have been capped at an altitude of $2.5 \mathrm{~km}$. In the case that a given lidar profile gets too noisy before this altitude, the profile is discarded and not taken into account for the statistics. This cutoff altitude is chosen in order to keep at least $75 \%$ of the profiles within the statistics (only $23 \%$ of the considered RL profiles reach $100 \%$ relative uncertainty at a height lower than $2.5 \mathrm{~km}$ ). This strategy simplifies the separate study of three atmospheric regions, defined as follows.

- Region (a) from ground to $180 \mathrm{~m}$ : no lidar data are available

- Region (b) from $180 \mathrm{~m}$ to $2.5 \mathrm{~km}$ : this is the only domain where there are lidar data. It is enclosed inside the dashed horizontal lines in Fig. 6.

- Region (c) from $2.5 \mathrm{~km}$ to $10 \mathrm{~km}$ : no lidar data are considered.

At first, a comparison of the absolute humidity profiles to the radiosonde profiles is performed. Unfortunately, only 18 valid clear sky radiosondes have been found during the periods where BASIL measured. In Fig. 6, the bias (on the left) and the standard deviation (on the right) to the RS are presented for the three cases: only-MWR, only-RL and the MWR+RL combination.

Region (a) exhibits the largest standard deviations (SDs) and biases, with similar values for the three cases. In addi- 

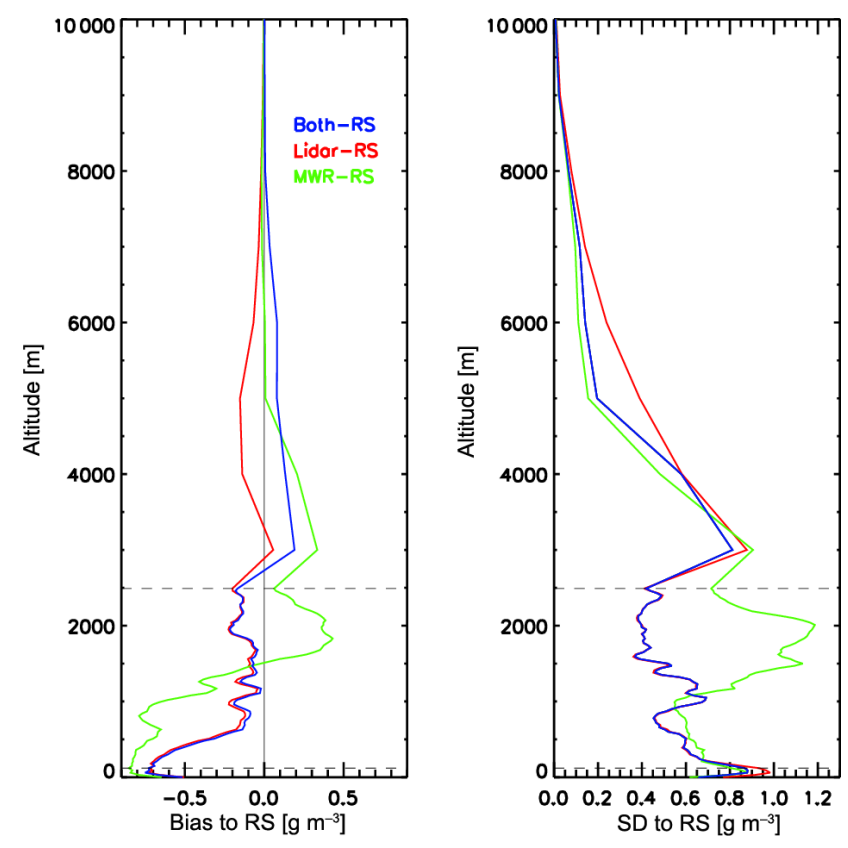

Figure 6. Mean and standard deviation of the difference between the 18 clear sky radiosondes: MWR (in green), RL (in red) and the combination of both (blue). The dashed horizontal lines enclose the region where the lidar data are used.

tion to the fact that no lidar data are available here, this result may be due to different surface-related local effects at the site where the RS was launched ( $\sim 4 \mathrm{~km}$ distance) and at the site where the instruments measure. In addition an insufficient venting of the RS in the lowest $100 \mathrm{~m}$ may act as an additional uncertainty.

In region (b), bias and standard deviation for the onlyRL and RL+MWR are very similar, whereby only-MWR reveals the largest values. The similarity between only-RL and the combination is again explained by the small uncertainty associated to the lidar measurements. The product of the combination tends to the lidar data when available, as seen in Sect. 4.1. From $\sim 500 \mathrm{~m}$ to $2.5 \mathrm{~km}$, both only-RL and RL+MWR show a small bias on the order of $\sim 0.2 \mathrm{~g} \mathrm{~m}^{-3}$, but below this altitude, the deviation increases up to $\sim 0.75 \mathrm{~g} \mathrm{~m}^{-3}$. This fact may suggest that the lidar data in the lower $500 \mathrm{~m}$ could have some additional issues with the RL OVF. This feature will be examined in more detail in Sect. 5.5.

In region (c) all the three values for the different retrievals are similar. The only-MWR seems to perform best when comparing to the RS, because both its bias and SD are the smallest. The only-RL case presents the largest bias and SD because in this region only information from the a priori is provided. The combination of the two sensors presents intermediate values, however, more similar to the only-MWR case.
Unfortunately, this set of only 18 radiosondes does not allow a significant assessment of the synergy benefits. In addition, when interpreting the results in Fig. 6, one must take into account that the RS itself presents some sources of uncertainty which are not easy to quantify, e.g., the launch distance of $4 \mathrm{~km}$ to the instrument site, drifts of the balloon, dry bias (Miloshevich et al., 2001). Because of that, other parameters are needed to further evaluate the synergy advantages. One quantity with this capability is the theoretical OEM uncertainty of the retrieved profiles (see Eq. 4). This parameter is studied in the following subsections.

\subsection{Theoretical error comparison}

As already mentioned in Sect. 3, the algorithm provides an estimation of the a posteriori error for the retrievals, see Eq. (4). For each profile the associated theoretical error profile is computed in the three different cases: using only-RL, only MWR and the RL+MWR combination.

In order to investigate the algorithm performance during day- and nighttime separately, Fig. 7 shows the mean theoretical errors for the three algorithm setups, differentiating between daytime and nighttime. Note that, in this study, no clipping is performed in the measurements, and thus, we cannot distinguish three regions according to lidar availability. This region separation will be used again later on.

Figure 7 presents the number of RL profiles reaching each specific altitude. Note that, for the sake of comparability, the theoretical error for each of the three retrieval cases has been averaged over the same number of profiles. As discussed in Sect. 2.1, the lidar performance is much better during nighttime, when more than $50 \%$ of the lidar data reach a maximum useful altitude of around $7 \mathrm{~km}$. The theoretical error during night is also lower than during daytime (i.e., about a factor of 3 smaller at an altitude of $4 \mathrm{~km}$ ), as expected. During daytime, the highest useful lidar height reaches only a maximum altitude of around $5.5 \mathrm{~km}$. In addition, only half of the profiles reach values higher than $3 \mathrm{~km}$. In these situations, the MWR information is expected to be a more powerful supplement to the lidar information. This is seen well in the improvement of the theoretical error due to the addition of the MWR information, which improves the theoretical error by approximately a $25 \%$ in the altitude range between 3 and $5 \mathrm{~km}$. The only-MWR case remains almost invariable because the instrument performs the same under different light conditions.

Another theoretical error analysis is performed clipping all lidar measurements at $2.5 \mathrm{~km}$, following the same argumentation as in Sect. 5.2. This way, the three previous atmospheric regions (a), (b) and (c) defined in Sect. 5.2 can be distinguished according to RL availability. Note that this simplification of the problem allows the relative impact of MWR and RL to be clearly specified in the different retrievals.

Figure 8 presents the a priori uncertainty, as well as an average over the 636 theoretical error profiles calculated after 


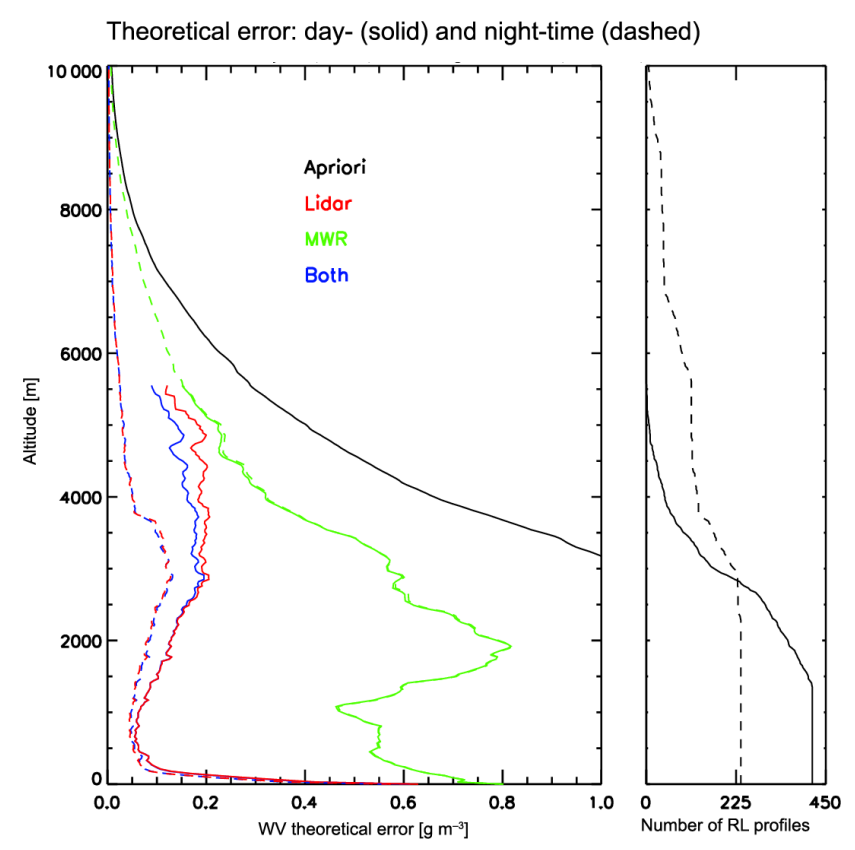

Figure 7. Left: mean theoretical error over the 636 clear sky cases during the complete HOPE period, separated into daytime (solid) and nighttime (dashed) measurements. In black: a priori uncertainty (lowest $3 \mathrm{~km}$ are out of margins). Red: only-RL. Green: only MWR. In blue: the MWR+RL. Right: number of RL profiles reaching each altitude, corresponding to the number of profiles used to calculate the average in the left panel.

running the OEM for all the HOPE clear sky periods. Clearly the uncertainty associated to the a priori is the largest, as it represents the atmospheric variability within the HOPE period. When only the TBs of the MWR are introduced in the algorithm, the average error estimate is reduced at least by half throughout the whole atmosphere with respect to the a priori uncertainty. When only the lidar information is used by the algorithm, the error in region (b) is strongly reduced with respect to the other two previous cases. Compared to the only-MWR error, which has an average of $\sim 0.7 \mathrm{~g} \mathrm{~m}^{-3}$, the only-RL is lowered to almost $0.1 \mathrm{~g} \mathrm{~m}^{-3}$. In regions (a) and (c) the only-RL error is larger than in region (b) because no lidar data are available and thus only the a priori information is used to complete the profile. The only-RL uncertainty is indeed especially large above $3 \mathrm{~km}$, where it tends to the a priori uncertainty, presenting larger values than the onlyMWR error.

However, when the combination of RL+MWR is performed, the resulting error is the smallest for all the altitudes. In region (b), the error is almost the same as for the onlyRL case. Outside this region, the MWR contribution plays an important role in reducing the uncertainty. In region (c), from average uncertainty values of 0.17 and $0.22 \mathrm{~g} \mathrm{~m}^{-2}$ for only-MWR and only-RL respectively, the uncertainty of the combination is reduced to an average value of $0.12 \mathrm{~g} \mathrm{~m}^{-2}$. Similarly, in the lowest region, the average error for the com- bination is 0.30 , in comparison to 0.71 and $0.33 \mathrm{~g} \mathrm{~m}^{-2}$ for the only-RL and only-MWR cases, respectively. In general, we can say that there is clear improvement in the theoretical error due to the synergy of the two instruments.

One can quantify the relative error reduction $\mathrm{err}_{\text {red }}$ of the joint retrieval in comparison to the instruments working alone. We can calculate this value as the difference between the single instrument and joint theoretical error profiles, divided by the single instrument one; that is

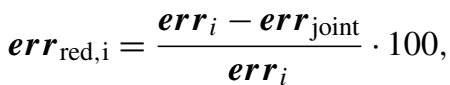

where $i=[\mathrm{RL}, \mathrm{MW}]$ and represents the averaged error profiles for the two different scenarios: when only-RL and onlyMWR is used (Fig. 8). Then, $\boldsymbol{e r} \boldsymbol{r}_{\text {red, }, i}$ is a profile representing a relative error reduction as a function of the altitude. The average error reduction for the absolute humidity in the complete atmospheric profile is $60 \%$ (38\%), with respect to the retrieval using only-MWR data (only-RL). This improvement is especially clear in region (c), above the available lidar data. The improvement of the combination in region (a) is better analyzed with the experiment in Sect. 5.5.

\subsection{Degrees of freedom}

Another parameter to assess the retrieval performance is the DOF (see Sect. 3.1). DOF allow us to study the amount of information provided by the different instruments in the three different atmospheric regions described in Sect. 5.2. Figure 9 represents the vertical profile of cumulative degrees of freedom $(\mathrm{CDOF})$ for the different instrument combinations, obtained as an average over 636 profiles. In the case of onlyMWR, the CDOF are smaller than for the other cases, reaching a maximum of 2.26 at $10 \mathrm{~km}$, in agreement with previous studies (Löhnert et al., 2007). Whenever lidar data are available, the CDOF increase linearly, due to the independent information of each altitude bin measured by the lidar. In the case of only-RL, above $2.5 \mathrm{~km}$ the cumulative DOF remain constant because no additional information is introduced. However, for the RL+MWR, the CDOF increase above $2.5 \mathrm{~km}$ thanks to the inclusion of the MWR measurements. Table 1 summarizes the values in Fig. 9. For the onlyRL case: in the regions where no lidar data are available (a and c), the DOF are, as expected, zero. In region (b), the total number of average DOF are around 26. This means that the lidar data, with the assumed $\mathbf{S}_{\mathrm{e}}$ and the constraint provided by $\mathbf{S}_{\mathrm{a}}$, provide $\sim 26$ independent pieces of information for humidity profile retrieval on average. The average total number of DOF in the column is largest for the combination of the two instruments, increasing by almost 2 DOF with respect to the only-RL case. The numbers for the MWR+RL combination show that the inclusion of MWR results mainly in an increase of DOF $(+1.6)$ in region (c), whereas in region (b) the DOF remain almost the same. This implies that large parts of the DOF contained in the only-MWR retrieval 


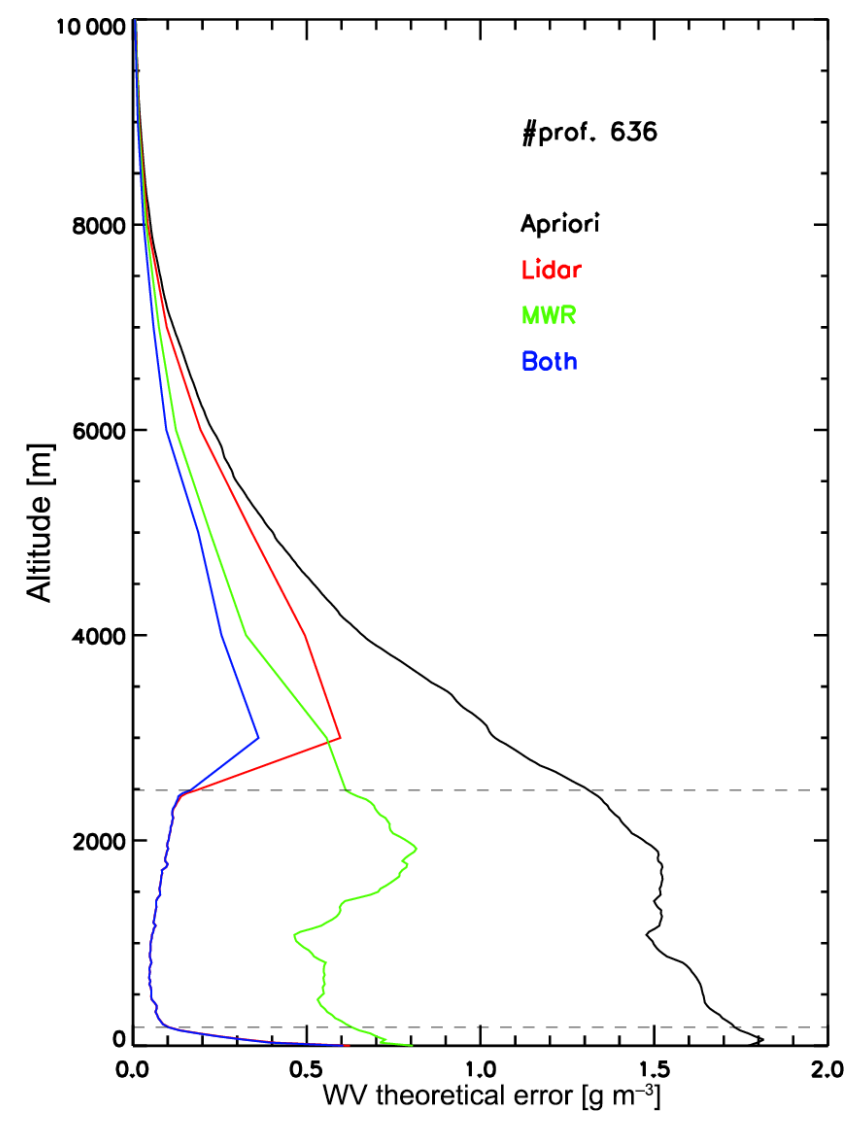

Figure 8. Mean theoretical error over 636 clear sky cases during the complete HOPE period. The lidar data have been artificially cut off at $2.5 \mathrm{~km}$. In black: a priori uncertainty. Red: only-RL. Green: only MWR. In blue: the MWR+RL. The dashed horizontal lines enclose the region where the lidar data are used.

for the complete profile (2.26) have now been shifted to the region above $2.5 \mathrm{~km}$. This optimal exploitation of the MWR information content due to constraints set by the lidar in other altitude regions clearly shows the synergy benefit.

\subsection{Sensitivity study 1: lower atmosphere}

As argued in Sect. 5.2, the high bias values for only-RL and RL+MWR from ground to $500 \mathrm{~m}$ (Fig. 6) might reveal a problem with the lidar OVF in this region. To assess the retrieval performance in the case of a larger non-overlap region, we run the retrieval considering that the OVF of the RL does not allow us to obtain valid measurements from the lowest $500 \mathrm{~m}$, instead of $18 \mathrm{~m}$. Thus, lidar data from 180 to $500 \mathrm{~m}$ are discarded in all the profiles. The algorithm is run again for the complete HOPE period, taking this condition into account and maintaining the clipping altitude at $2.5 \mathrm{~km}$ as described in Sect. 5.2.

Figure 10 shows the mean theoretical error for the expanded zero overlap region (ZOR) together with the initial ZOR (up to $180 \mathrm{~m}$ ). In both cases (initial ZOR and increased

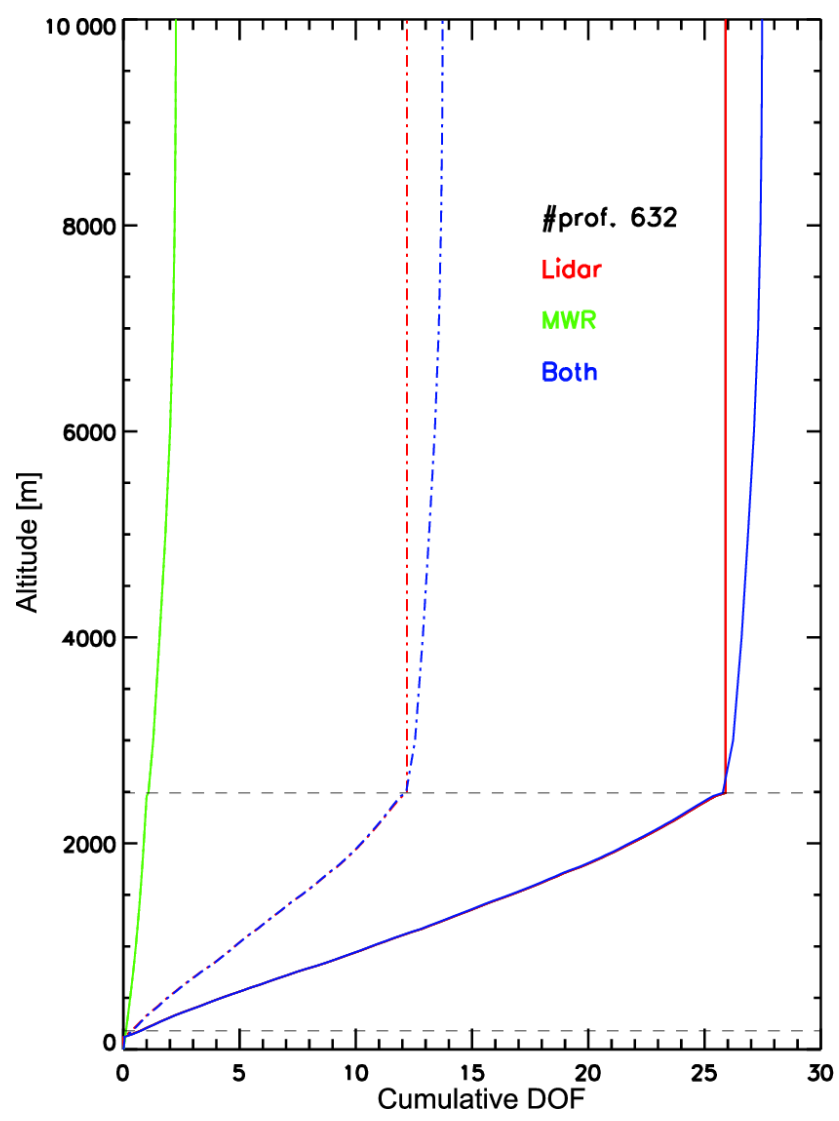

Figure 9. Cumulative degrees of freedom per profile for the different instrument combinations: in red, only-RL; in green, only-MWR and in blue, MWR+RL. The dotted-dashed lines represent the degrees of freedom for the case where the RL uncertainty has been multiplied by 4 . The average number of DOF in every region are summarized on Table 1. The dashed horizontal gray lines enclose the part of the atmosphere where lidar data have been considered. The number of elements in the measurement and state vectors are 77 (66 for the dashed case) and 91, respectively.

ZOR), the results are very similar in regions where the RL data are available (from $500 \mathrm{~m}$ to $2.5 \mathrm{~km}$ ), with the theoretical error of the MWR+RL matching that of the onlyRL. However, in the lower region of the increased ZOR, the $M W R+R L$ error is smaller than the only-RL case: there is an uncertainty reduction at the ground level of about $0.1 \mathrm{~g} \mathrm{~m}^{-3}$, which is gradually reduced towards the region where RL data are available. This result nicely shows the synergy benefit of both instruments in the atmosphere below $500 \mathrm{~m}$. Above this point and up to $2.5 \mathrm{~km}$, the error is almost equal for the cases of initial ZOR and increased ZOR. From 2.5 to $10 \mathrm{~km}$, the increased ZOR shows a slight increase in theoretical error of $\sim 0.05$ and $\sim 0.02 \mathrm{~g} \mathrm{~m}^{-3}$ for the RL+MWR and onlyRL cases, with respect to the initial ZOR. This is because the MWR information content is redistributed and more efficiently used in the lower layers of the atmosphere. 
Table 1. Degrees of freedom for signal comparison for absolute humidity. Average over 636 profiles. The atmosphere is separated into three regions according to lidar availability. The DOF are presented for three cases: only RL, only MWR and the combination of both instruments. In the upper part, no increment on the RL uncertainty has been considered. In the bottom part, the RL uncertainty has been multiplied by a factor of 4 .

\begin{tabular}{llll}
\hline Region & RL & MWR & Combination \\
\hline (a) Ground to $180 \mathrm{~m}$ & 0.00 & 0.07 & 0.03 \\
(b) $180 \mathrm{~m}$ to $2.5 \mathrm{~km}$ & 25.90 & 1.01 & 25.75 \\
(c) 2.5 to $10 \mathrm{~km}$ & 0.00 & 1.18 & 1.69 \\
\hline Total & 25.90 & 2.26 & 27.47 \\
\hline (a) Ground to $180 \mathrm{~m}$ & 0.00 & 0.07 & 0.06 \\
(b) $180 \mathrm{~m}$ to $2.5 \mathrm{~km}$ & 12.19 & 1.01 & 12.11 \\
(c) 2.5 to $10 \mathrm{~km}$ & 0.00 & 1.18 & 1.57 \\
\hline Total & 12.19 & 2.26 & 13.74 \\
\hline
\end{tabular}

\subsection{Sensitivity study 2: increase of the RL error}

In Sect. 3.3 the components of the covariance matrix $\mathbf{S}_{\mathrm{e}}$ have been determined to our best knowledge. However, it might be possible that additional uncertainty sources exist. In order to better understand the impact of the lidar uncertainties, we performed a sensitivity study increasing the lidar uncertainty.

The magnitude of the increase in RL measurement uncertainty is chosen based on the discrepancy between the theoretical error $\left(0.1 \mathrm{~g} \mathrm{~m}^{-3}\right.$, Fig. 8$)$ and the mean deviation to the $\mathrm{RS}\left(0.4 \mathrm{~g} \mathrm{~m}^{-3}\right.$, Fig. 6$)$ at around $2 \mathrm{~km}$, showing that the deviation to the RS is 4 times larger than the originally assumed error. Therefore, we have increased the RL uncertainty by a factor of 4 to study the sensitivity of the retrieved profile error with respect to the RL measurement uncertainty. Note that also in this experiment the three regions (a), (b) and (c) as defined in Sect. 6 are valid.

The results of this test are plotted in Fig. 10, together with the initial values (without increment), for the only-RL and MWR+RL cases. The new averaged errors are very similar at the ground, but they have increased by a factor of 2 to 3 in region (b). The uncertainty is less than a factor of 4 because of the stabilization by the prior. In case of increased RL uncertainties, the difference between the errors of the only-RL and RL+MWR (dashed lines) is more noticeable than in the original case (solid line), especially from $2 \mathrm{~km}$ upwards. Note that already at $2.5 \mathrm{~km}$, the error reduction for including the MWR, reaches values close to $0.1 \mathrm{~g} \mathrm{~m}^{-3}$. Thus, as expected the synergy benefit increases.

In addition, when an increment in the RL uncertainty is considered, the amount of useful information provided by this instrument is smaller, and thus the DOF are reduced. This reduction can been seen in all regions where the RL is involved (Fig. 9). In the case of an uncertainty increase of a factor of 4 , the total average DOF are reduced by a factor

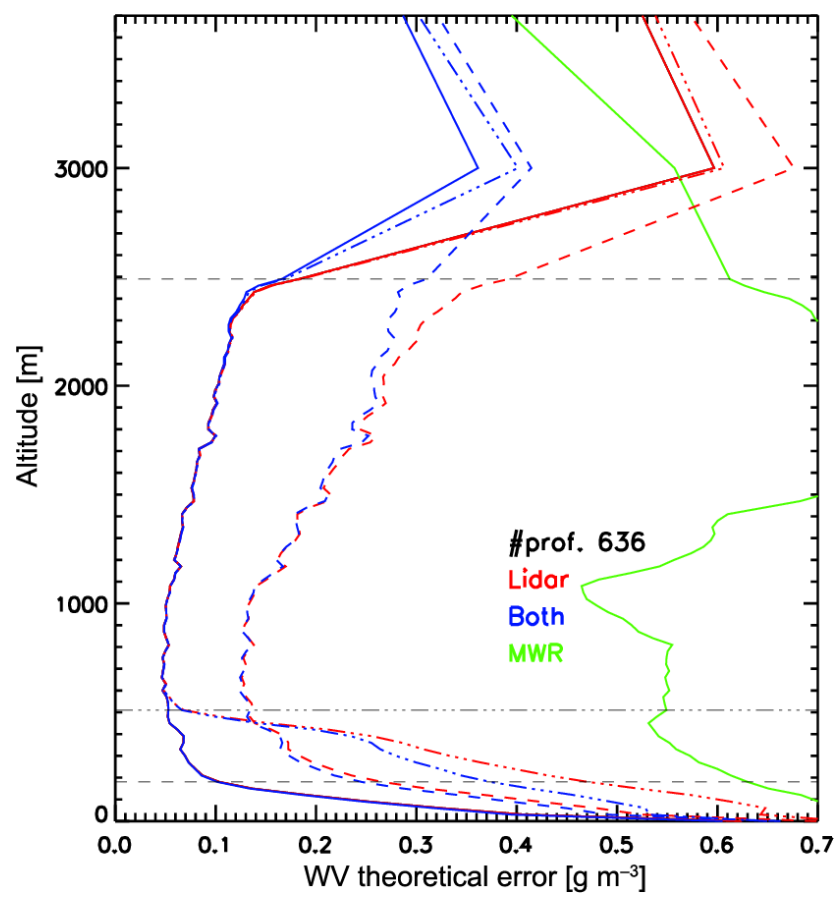

Figure 10. Mean theoretical error over 636 clear sky cases during the complete HOPE period. Red: only RL has been introduced in the algorithm. Green: only-MWR. In blue, the combination of RL and MWR. The dashed horizontal black lines define the region where lidar data have been considered available. The dashed red and blue lines represent the result when the lidar uncertainty has been incremented by a factor of 4 . The dotted-dashed red and blue lines correspond to the case where lidar data have been suppressed from ground until $500 \mathrm{~m}$. Solid lines show the errors without increments, as shown in Fig. 8.

of $\sim 2$ (Table 1). Note that, naturally, the DOF values for the MWR only retrieval remain the same.

The results presented so far confirm that the RL+MWR water vapor synergy is meaningful and advantageous. In addition, they suggest that a careful specification of the instrument uncertainties, especially for the RL, is required.

\section{Conclusions}

Atmospheric humidity is an essential variable for the description of any meteorological process. Highly resolved, accurate and continuous measurements of this parameter are required for a deeper understanding of many atmospheric phenomena. However, nowadays there is no single instrument that can provide all of the following requirements simultaneously: complete vertical coverage, high vertical and temporal resolution of the atmospheric humidity profiles and satisfactory performance under all weather conditions. This is why the synergy of different sensors has come more and more into focus in the last years. 
In this paper, we present a new and robust method to combine water vapor mixing ratio Raman lidar profiles and multifrequency brightness temperatures from a microwave radiometer. The joint algorithm that combines the two sensors is based on an optimal estimation method, and can be also applied to measurements from one instrument alone. Results for $53 \mathrm{~h}$ of clear sky measurements during the HOPE period are presented for absolute humidity profile retrievals.

The improvements of merging both instrument systems have been consistently analyzed in terms of both the reduction of the theoretical error and the increase of DOF. Significant advantages of instrument synergy are clearly shown above the highest valid lidar signal. For example, when applying the combined retrieval to the complete HOPE period, the absolute humidity theoretical error above $\sim 3 \mathrm{~km}$ is reduced by a factor of 2 with respect to the case where only lidar data are used. The addition of the MWR information to the RL results in 1.6 additional degrees of freedom for signal, which are mainly distributed in the layers above the lidar noise threshold. The synergy presents its strongest advantages in the regions where RL data are not available, whereas in the regions where both instruments are available, RL dominates the retrieval.

With the expansion of the ground-based network of atmospheric profiling stations, the application of the OEM at several sites under different climate conditions will become possible. In this respect, the definition of an appropriate background uncertainty covariance needs to be carefully addressed. Further studies will extend the algorithm to cloudy cases and to temperature and relative humidity profiling. In addition, the method will be applied, not only to ground-based measurements, but also to airborne data (Mech et al., 2014), which will allow the study of meteorological phenomena to be completed from the airborne point of view.

\section{Data availability}

The data used in this study are available at the $\mathrm{HD}(\mathrm{CP})^{2}$ Data Archive (Stamnas et al., 2016), which is freely accessible by all users from the $\mathrm{HD}(\mathrm{CP})^{2}$ Web Portal (University of Hamburg, 2016). The details for the data structure and organization are also found at Stamnas et al. (2016).

Acknowledgements. Acknowledgements: This research has been financed by ITARS (www.itars.net), the European Union Seventh Framework Programme FP7: People, ITN Marie Sklodowska Curie Actions Programme under grant agreement no. 289923. The authors would like to acknowledge the Federal Ministry of Education and Research in Germany (BMBF), who, through the research programme High Definition Clouds and Precipitation for Climate Prediction $\mathrm{HD}(\mathrm{CP})^{2}$, financed HOPE. Special thanks to Kerstin Ebell (for her important contribution to the early stages of the project), Dave Turner (for his always fruitful ideas) and Bjorn Stevens (for his useful advice).
Edited by: T. Islam

Reviewed by: D. Cimini, A. H. Haefele, and two anonymous referees

\section{References}

Adam, S., Schwitalla, T., Hammann, E., Behrendt, A., and Wulfmeyer, V.: Composite temperature profiles from Raman Lidar and Microwave Radiometer, in: 27th International Laser Radar Conference, 2015 New York, 2015.

Althausen, D., Müller, D., Ansmann, A., Wandinger, U., Hube, H., Clauder, E., and Zörner, S.: Scanning 6-wavelength 11-channel aerosol lidar, J. Atmos. Ocean. Tech., 17, 1469-1482, 2000.

Behrendt, A., Wulfmeyer, V., Hammann, E., Muppa, S. K., and Pal, S.: Profiles of second- to fourth-order moments of turbulent temperature fluctuations in the convective boundary layer: first measurements with rotational Raman lidar, Atmos. Chem. Phys., 15, 5485-5500, doi:10.5194/acp-15-5485-2015, 2015.

Bevis, M., Businger, S., Herring, T. A., Rocken, C., Anthes, R. A., and Ware, R. H.: GPS meteorology: Remote sensing of atmospheric water vapor using the global positioning system, J. Geophys. Res.-Atmos., 97, 15787-15801, doi:10.1029/92JD01517, 1992.

Bianco, L., Cimini, D., Marzano, F., and Ware, R.: Combining microwave radiometer and wind profiler radar measurements for high-resolution atmospheric humidity profiling, J. Atmos. Ocean. Tech., 22, 949-965, 2005.

Brocard, E., Philipona, R., Haefele, A., Romanens, G., Mueller, A., Ruffieux, D., Simeonov, V., and Calpini, B.: Raman Lidar for Meteorological Observations, RALMO - Part 2: Validation of water vapor measurements, Atmos. Meas. Tech., 6, 1347-1358, doi:10.5194/amt-6-1347-2013, 2013.

Crewell, S. and Löhnert, U.: Accuracy of cloud liquid water path from ground-based microwave radiometry 2. Sensor accuracy and synergy, Radio Sci., 38, 8042, doi:10.1029/2002RS002634, 2003.

Delanoe, J. and Hogan, R. J.: A variational scheme for retrieving ice cloud properties from combined radar, lidar, and infrared radiometer, J. Geophys. Res.-Atmos., 113, doi:10.1029/2007JD009000, 2008.

Di Girolamo, P., Marchese, R., Whiteman, D. N., and Demoz, B. B.: Rotational Raman lidar measurements of atmospheric temperature in the UV, Geophys. Res. Lett., 31, 101106, doi:10.1029/2003GL018342, 2004.

Di Girolamo, P., Summa, D., and Ferretti, R.: Multiparameter Raman Lidar Measurements for the Characterization of a Dry Stratospheric Intrusion Event, J. Atmos. Ocean. Tech., 26, 17421762, doi:10.1175/2009JTECHA1253.1, 2009.

Di Girolamo, P., Summa, D., Bhawar, R., Di Iorio, T., Cacciani, M., Veselovskii, I., Dubovik, O., and Kolgotin, A.: Raman lidar observations of a Saharan dust outbreak event: Characterization of the dust optical properties and determination of particle size and microphysical parameters, Atmos. Environ., 50, 66-78, doi:10.1016/j.atmosenv.2011.12.061, 2012.

Dinoev, T., Simeonov, V., Arshinov, Y., Bobrovnikov, S., Ristori, P., Calpini, B., Parlange, M., and van den Bergh, H.: Raman Lidar for Meteorological Observations, RALMO - Part 
1: Instrument description, Atmos. Meas. Tech., 6, 1329-1346, doi:10.5194/amt-6-1329-2013, 2013.

Dunbar, T., Barlow, J., and Belcher, S.: An Optimal Inverse Method Using Doppler Lidar Measurements to Estimate the Surface Sensible Heat Flux, Bound.-Lay. Meteorol., 150, 49-67, 2014.

Ebell, K., Orlandi, E., Hünerbein, A., Löhnert, U., and Crewell, S.: Combining ground-based with satellite-based measurements in the atmospheric state retrieval: Assessment of the information content, J. Geophys. Res.-Atmos., 118, 6940-6956, doi:10.1002/jgrd.50548, 2013.

Foth, A., Baars, H., Di Girolamo, P., and Pospichal, B.: Water vapour profiles from Raman lidar automatically calibrated by microwave radiometer data during HOPE, Atmos. Chem. Phys., 15, 7753-7763, doi:10.5194/acp-15-7753-2015, 2015.

Furumoto, J., Kurimoto, K., and Tsuda, T.: Continuous observations of humidity profiles with the MU radar-RASS combined with GPS and radiosonde measurements, J. Atmos. Ocean. Tech., 20, 23-41, 2003.

Gendt, G., Dick, G., Reigber, C., Tomassini, M., Liu, Y., and Ramatschi, M.: Near Real Time GPS Water Vapor Monitoring for Numerical Weather Prediction in Germany, J. Meteorol. Soc. Japan. Ser. II, 82, 361-370, 2004.

Han, Y., Westwater, E. R., and Ferrare, R. A.: Applications of Kalman Filtering to Derive Water Vapor Profiles from Raman Lidar and Microwave Radiometers, J. Atmos. Ocean. Tech., 14, 480-487, doi:10.1175/15200426(1997)014<0480:AOKFTD>2.0.CO;2, 1997.

Janssen, M.: Atmospheric remote sensing by microwave radiometry, Wiley-interscience publication, Wiley, New York, 1993.

Löhnert, U. and Crewell, S.: Accuracy of cloud liquid water path from ground-based microwave radiometry 1. Dependency on cloud model statistics, Radio Sci., 38, 8041, doi:10.1029/2002RS002654, 2003.

Löhnert, U. and Maier, O.: Operational profiling of temperature using ground-based microwave radiometry at Payerne: prospects and challenges, Atmos. Meas. Tech., 5, 1121-1134, doi:10.5194/amt-5-1121-2012, 2012.

Löhnert, U., Crewell, S., and Simmer, C.: An integrated approach toward retrieving physically consistent profiles of temperature, humidity, and cloud liquid water, J. Appl. Meteorol., 43, 12951307, 2004.

Löhnert, U., van Meijgaard, E., Baltink, H. K., Groß, S., and Boers, R.: Accuracy assessment of an integrated profiling technique for operationally deriving profiles of temperature, humidity, and cloud liquid water, J. Geophys. Res.-Atmos., 112, doi:10.1029/2006JD007379, 2007.

Löhnert, U., Turner, D. D., and Crewell, S.: Ground-Based Temperature and Humidity Profiling Using Spectral Infrared and Microwave Observations. Part I: Simulated Retrieval Performance in Clear-Sky Conditions, J. Appl. Meteorol. Climatol., 48, 10171032, doi:10.1175/2008JAMC2060.1, 2009.

Löhnert, U., Schween, J. H., Acquistapace, C., Ebell, K., Maahn, M., Barreraverdejo, M., Hirsikko, A., Bohn, B., Knaps, A., O'connor, E., Simmer, C., Wahner, A., and Crewell, S.: JOYCE: Jülich Observatory for Cloud Evolution, B. Am. Meteorol. Soci., 96, 1157-1174, doi:10.1175/BAMS-D-14-00105.1, 2014.

Maschwitz, G., Löhnert, U., Crewell, S., Rose, T., and Turner, D. D.: Investigation of ground-based microwave radiometer calibra- tion techniques at $530 \mathrm{hPa}$, Atmos. Meas. Tech., 6, 2641-2658, doi:10.5194/amt-6-2641-2013, 2013.

Mech, M., Orlandi, E., Crewell, S., Ament, F., Hirsch, L., Hagen, M., Peters, G., and Stevens, B.: HAMP - the microwave package on the High Altitude and LOng range research aircraft (HALO), Atmos. Meas. Tech., 7, 4539-4553, doi:10.5194/amt7-4539-2014, 2014.

Miloshevich, L. M., Vömel, H., Paukkunen, A., Heymsfield, A. J., and Oltmans, S. J.: Characterization and correction of relative humidity measurements from Vaisala RS80-A radiosondes at cold temperatures, J. Atmos. Ocean. Tech., 18, 135-156, 2001.

Povey, A. C., Grainger, R. G., Peters, D. M., and Agnew, J. L.: Retrieval of aerosol backscatter, extinction, and lidar ratio from Raman lidar with optimal estimation, Atmos. Meas. Tech., 7, 757776, doi:10.5194/amt-7-757-2014, 2014.

Rodgers, C.: Inverse Methods for Atmospheric Sounding: Theory and Practice, World Scientific Publishing Company, Incorporated, Singapore, 2000.

Rose, T., Crewell, S., Löhnert, U., and Simmer, C.: A network suitable microwave radiometer for operational monitoring of the cloudy atmosphere, Atmos. Res., 75, 183-200, doi:10.1016/j.atmosres.2004.12.005, 2005.

Schneebeli, M.: Advancements in Ground-Based Microwave Remote Sensing of the Troposphere - Calibration, Data Retrieval and Applications, Ph.D. thesis, Philosophisch Naturwissenschaftliche Fakultät, Universität Bern, Bern, Switzerland, 2009.

Sica, R. J. and Haefele, A.: Retrieval of temperature from a multiple-channel Rayleigh-scatter lidar using an optimal estimation method, Appl. Opt., 54, 1872-1889, doi:10.1364/AO.54.001872, 2015.

Sica, R. J. and Haefele, A.: Retrieval of water vapor mixing ratio from a multiple channel Raman-scatter lidar using an optimal estimation method, Appl. Opt., 55, 763-777, doi:10.1364/AO.55.000763, 2016.

Stamnas, E., Lammert, A., Winkelmann, V., and Lang, U.: The $\mathrm{HD}(\mathrm{CP})^{2}$ Data Archive for Atmospheric Measurement Data, ISPRS Int. J. Geo-Inf., 5, 124 pp., http://www.mdpi.com/ 2220-9964/5/7/124, 2016.

Stankov, B. B.: Multisensor Retrieval of Atmospheric Properties, B. Am.Meteorol. Soc., 79, 1835-1854, doi:10.1175/15200477(1998)079<1835:MROAP>2.0.CO;2, 1998.

Steinke, S., Eikenberg, S., Löhnert, U., Dick, G., Klocke, D., Di Girolamo, P., and Crewell, S.: Assessment of small-scale integrated water vapour variability during HOPE, Atmos. Chem. Phys., 15, 2675-2692, doi:10.5194/acp-15-2675-2015, 2015.

Stevens, B. and Bony, S.: Water in the atmosphere, Phys. Today, 66, p. 29, doi:10.1063/PT.3.2009, 2013.

Turner, D. D., Clough, S. A., Liljegren, J. C., Clothiaux, E. E., Cady-Pereira, K. E., and Gaustad, K. L.: Retrieving Liquid Water Path and Precipitable Water Vapor From the Atmospheric Radiation Measurement (ARM) Microwave Radiometers, IEEE T. Geosci. Remote, 45, 3680-3690, doi:10.1109/TGRS.2007.903703, 2007.

University of Hamburg: The $\mathrm{HD}(\mathrm{CP})^{2}$ Portal, available at: http:// icdc.zmaw.de/1/projekte/hdcp2.html, last access: August 2016.

Whiteman, D. N.: Examination of the traditional Raman lidar technique. I. Evaluating the temperature-dependent lidar equations, Appl. Opt., 42, 2571-2592, doi:10.1364/AO.42.002571, 2003. 
Wilks, D. S.: Statistical Method in the Atmospheric Sciences, Interational Geophysics Series, Elsevier, United States of America, 2006.
Wulfmeyer, V., Bauer, H.-S., Grzeschik, M., Behrendt, A., Vandenberghe, F., Browell, E. V., Ismail, S., and Ferrare, R. A.: Fourdimensional variational assimilation of water vapor differential absorption lidar data: The first case study within IHOP_2002, Mon. Weather Rev., 134, 209-230, 2006. 